\title{
Articles
}

\section{Online Marriage Education During COVID-19 Home Lockdown: A Multiple- Baseline Single-Case Experimental Design}

\author{
Jennifer Harley Chalmers*a
}

[a] Independent Researcher, Pasig City, Philippines.

\begin{abstract}
Flexible-delivery marriage education (ME) has many advantages over traditional alternatives in reaching couples during the time of COVID-19 lockdown or other national emergencies. In an effort to add to the research of flexible-delivery ME, this exploratory study evaluated an online class adapted from an empirically-validated, marriage curriculum (Four Gifts of Love Class, [FGL]) under home lockdown conditions lasting over 2 months caused by COVID-19 government restrictions. Using a concurrent multiple-baseline single-case experimental design, three distressed couples residing in the Philippines completed seven online lessons over 7 weeks while experiencing home lockdown. Visual analysis of the data suggested that all three couples responded positively to the intervention. The Tau-U and $S M D a l l$ analyses for each couple ranged from a small to large effect size on measures of marital adjustment (weighted average Tau- $U$ $=.50, p<.05 ; \mathrm{BC}-\mathrm{SMD}=0.34$ ) and romantic love (weighted average Tau-U $=.52, p<.01 ; \mathrm{BC}-\mathrm{SMD}=0.31$ ), with increases reaching clinical and statistical significance for one couple out of the three. In addition, there was no attrition. The promising results from this preliminary study suggested that the online adaptation of FGL as a flexible-delivery ME could mitigate marital decline, especially during times of calamity when traditional-delivery ME is unavailable and marital decline is predicted. Further study of this program and other online ME programs are recommended to expand the limited research in this area of flexible-delivery ME.
\end{abstract}

Keywords: COVID-19, online-marriage education, romantic love, marital adjustment, marital stress, multiple-baseline design

Interpersona, 2020, Vol. 14(2), 150-168, https://doi.org/10.5964/ijpr.v14i2.3971

Received: 2020-07-05. Accepted: 2020-10-16. Published (VoR): 2020-12-22

*Corresponding author at: 1906 Orient Square Building, 1605 Pasig City, Philippines. E-mail: jenniferc@fourgiftsoflove.org

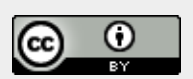

This is an open access article distributed under the terms of the Creative Commons Attribution 4.0 International License, CC BY 4.0 (https://creativecommons.org/licenses/by/4.0/), which permits unrestricted use, distribution, and reproduction in any medium, provided the original work is properly cited.

At the start of 2020, many marriages across the world experienced external stressors brought on by novel coronavirus (COVID-19). Worldwide calamity invaded the lives of couples resulting in sudden economic and social distress as many governments reacted with protective measures (National Economic and Development Authority, 2020; United Nations, 2020).

In the Philippines, home lockdown, or Enhanced Community Quarantine, started mid-March, 2020. Living restrictions were imposed with strict stay-at-home orders, only allowing exceptions for purchasing food and attending to medical needs (Medialdea, 2020a, 2020b). This instant-life disruption was periodically extended for a total of 10 weeks for certain regions until the quarantine was only moderately lifted in stages during the following months (Duque, 2020). Families were forced to make sudden decisions and adjustments due to school closure, restricted travel, furloughed jobs without pay, unavailable domestic-support/child-care, health safety, work-at-home jobs, and limited medical resources (National Economic and Development Authority, 2020). Although data on the effects of this particular quarantine period has not yet been formally researched, a 
review of 24 studies on the topic of the psychological impact from past quarantine measures would suggest a correlation between length of quarantine and negative emotional effects (Brooks et al., 2020).

With the life disruptions that have accompanied strict stay-at-home orders, it may be expected that couples would experience the same marital distress as research during past calamities has suggested (Cohan \& Cole, 2002; Lau et al., 2005; Peacock, Morrow, \& Gladwin, 1997). A report by Cohan and Cole (2002) indicated a significant divorce rate increase during 1990 for those residing in the areas hardest hit by Hurricane Hugo in 1989 , which was more than double that of the state-wide divorce rate. Peacock et al. (1997) studied the effects of Hurricane Andrew that devastated southern Florida in 1992 and found that $56.1 \%$ of the respondents $(N=$ 504) in South Dade County reported heightened stress with their relationship.

Research from another major-life disruption, the SARS epidemic in 2003, also offers predictions about the potential effects of COVID-19 related stressors on marital satisfaction. In a survey of over 800 Hong Kong residents during SARS, Lau et al. (2005) reported 16\% experiencing posttraumatic symptoms and approximately $40 \%$ experiencing elevated family stress due to SARS-related disruptions. With the increase of mental health issues and family stressors, marital satisfaction declined, as indicated by divorce rates in Hong Kong increasing by $20 \%$ in 2004 when compared to 2002 (Census and Statistics Department, Hong Kong, 2018).

A 2-year longitudinal study by Bodenmann (1997) studied the influences of external stressors on relationship quality. The report indicated a correlation between increases of external stressors and decreases in couple satisfaction, $F(1,47)=3.1, p<.08$. In addition, through logistic regression, a prediction of relationship outcome after 2 years demonstrated an accurate classification of stability or instability for $98.2 \%$ of the cases. Two primary predictors for relationship instability were verbal negativity and internalization of anger. The conclusion was that a couple's ability to dyadically cope when stressors arise led to improved relationship satisfaction and stability.

Specifically concerning COVID-19, Balzarini et al. (2020) studied the relationship between COVID-19 related stressors and relationship quality with online surveys collected from 57 countries $(N=3,593)$. They found that lower relationship quality was associated with increased external stress resulting from COVID-19. As a conclusion, they suggested ways to mitigate the potential marital decline with the following recommendation:

We demonstrate that perceived partner responsiveness-the extent to which people believe that their partners understand, validate, and care for them-in relationships may confer protective "armor" against spillover of these stressors into their relationship and may help people maintain better quality relationships in the face of stress (p. 23).

One objective of marriage education (ME) has been to teach couples relationship skills, including dyadic problem solving, positive communication, and partner responsiveness to proactively mitigate marital decline (Larson, 2004). However, traditional-delivery marriage education (i.e., educational materials delivered in a faceto-face, group-format setting with an educator present) would not be feasible under these natural-disaster or health-epidemic circumstances due to understandable engagement hurdles (e.g., home lockdown). A possible solution to these engagement hurdles during a time of calamity would be to offer flexible-delivery modalities.

In recent years, ME developers have been encouraged to create innovative and flexible-delivery methods, such as self-study (education materials provided with no educator present) and blended-delivery formats (self-study and traditional components) as a way to proactively reach and engage couples (Larson, 2004; McAllister, 
Duncan, \& Hawkins, 2012). Although self-study methods may have the highest potential of decreasing typical engagement hurdles (e.g., cost, schedule, distance), there is concern regarding equal effectiveness as compared to traditional-delivery ME.

A meta-analysis by McAllister et al. (2012) compared 103 traditional, self-study, and blended-delivery programs within marriage and relationship education (MRE). For aggregate marriage quality measures, the results indicated that the traditional programs on average had a lower effect size $(d=0.252, p<.001, k=77)$ than blended programs $(d=0.429, p<.01, k=13)$; and self-study programs had the smallest effect size $(d=0.032$, $n s, k=13)$. In other words, self-study programs had a low practical effect on improving marriage quality as demonstrated by the effect-size data (effect size reference, "small: $d=0.20$, medium: $d=0.50$, large: $d=0.80$ "; Cohen, 1988, p. 40).

Regardless of the statistically insignificant and small practical effect on marriage quality resulting from selfstudy programs, McAllister et al. (2012) predicted a future increase of self-study, online-delivery options as a way of reaching additional demographics. Two of the 13 , self-study programs identified in their meta-analysis involved an online format with married couples and were found to be effective in improving marital satisfaction (Duncan, Steed, \& Needham, 2009; Kalinka, Fincham, \& Hirsch, 2012).

Duncan et al. (2009) compared two formats of an ME program: 1) traditional (6, 2-hour workshops led by an accountability coach); and 2) web-based (reading/exercises on a set schedule with no accountability). The results found no significant difference in relationship-satisfaction scores (RELATE) between the traditional format compared to the web-based format; and both demonstrated significant gains over the control group, $F(2,40)=$ 4.99, $p=.012$. Attrition was about the same for all conditions (web-based: $35 \%$; traditional: $37 \%$; and control: $33 \%$ ). For the online intervention, the aggregate effect size for marriage quality was $d=0.355$ according to McAllister et al. (2012). When the effect size for relationship satisfaction, in particular, was calculated with the data provided, the effect size was small ( $d_{\mathrm{ppc} 2}=0.263$, Lenhard \& Lenhard, 2016; Morris, 2008).

Kalinka et al. (2012) studied the effects of an online ME for expectant couples. The program consisted of 12 , online-assignment modules across 2 months with biweekly reminders to complete the assignments and appreciation messages for participation. The attrition was $46 \%$ for the online group and $13 \%$ for the placebo-control group. The aggregate effect size for marriage quality was insignificant $(d=0.026)$ according to McAllister et al. (2012). The effect size for relationship satisfaction (CSI) was small $\left(g_{\text {Hedges }}=0.24, p<.005\right)$.

In a recent publication, Doss et al. (2016) adapted an empirically-based, in-person couple therapy curriculum to an online format. The program involved distressed couples ( $80 \%$ married) completing 7 hours of online reading and exercises with four, 15-minute, coaching-telephone sessions. When compared to the waitlist control group, the effect size for relationship satisfaction (CSI-4) was medium $\left(d_{\text {Cohen }}=0.69, p<.001\right)$. The attrition for the online group was $15 \%$.

The limited empirical research within self-directed, online-delivery ME suggested a range of effect-sizes for marriage-satisfaction measures and attrition concerns that could discourage ME developers; yet there appears to be a demand for it as Georgia and Doss (2013) identified. In a survey of 1,160 individuals, these researchers asked about educational resources that helped their romantic relationships. Self-help resources were identified as more helpful than couple therapy. When asked about resources they would use in the future, web-based, structured relationship education with feedback was the preferred delivery format. The Pew Research Center 
confirmed the results of Georgia and Doss (2013) by reporting the rapid growth of Internet availability across demographics and an increase of those seeking online, self-help information (Anderson, 2019; Anderson, Perrin, Jiang, \& Kumar, 2019; Silver \& Huang, 2019).

As more ME developers are becoming aware of this need, McAllister et al. (2012) recommended that existing empirically-validated programs consider creating self-study options with set schedules to complete homework assignments and accountability components (e.g., weekly phone call by an educator) to help lower attrition and heighten efficacy. Overall, online-delivery, self-study ME programs with accountability seem to offer a promising direction for overcoming engagement hurdles, especially during major life disruptions when couples need it the most.

In an attempt to add to the limited research of online ME programs and take the advice of McAllister et al. (2012) to adapt existing empirically-validated programs, this current study explored the effectiveness of an online marriage curriculum (Four Gifts of Love Class Online [FGLO]). FGLO utilized a set homework schedule with weekly accountability elements to address attrition and was adapted from the empirically-based, blended-format Four Gifts of Love Class (FGL). The FGL framework was chosen due to its focus on sustaining the feeling of romantic love as the primary ME goal and demonstrating a large effect size for improvements in both marital adjustment $\left(d_{\mathrm{ppc} 2}=1.11\right.$, Marital Adjustment Test [MAT]; Locke \& Wallace, 1959) and romantic love $\left(d_{\mathrm{ppc} 2}=0.68\right.$, Love Bank Inventory [LBI]; Harley, 2010), with no attrition (Chalmers, 2019).

The aim of the current research was to provide a preliminary study of FGLO with the primary research question: Does participating in FGLO improve marital adjustment and romantic love in marriage? It was predicted that the measures would show improvements within the FGLO phase as compared to Baseline, similar to the large effect size demonstrated by FGL. Also, a second question as related to engagement was: Will the couples complete FGLO? It was predicted that there would be no attrition.

It should be mentioned that the original intent of the study was to evaluate this curriculum under normal living conditions. However, the current world circumstances created a unique opportunity to study FGLO during distressing times. Regardless of these new conditions, the aim, research questions, predictions, and method remained the same.

\section{Method}

\section{Design}

A concurrent multiple-baseline single-case experimental design across couples was used, where the start of intervention was staggered across time (Kazdin, 2011). This design allows comparisons between nontreatment and treatment phases across and within subjects. Three married couples $(n=6)$ were randomly assigned to a 2-month or 10-day baseline before accessing intervention (Class, FGLO).

\section{Participants}

In an attempt to recruit couples from different demographics and nationalities, as well as those who may not be interested in or able to attend a traditional- or blended-delivery ME due to engagement hurdles (e.g., schedule, child care, location), private invitations to participate in an online-delivery marriage class were offered 
by members from two, Protestant churches in the Philippines. At baseline, three heterosexual, married couples residing in the National Capital Region (NCR) region of the Philippines volunteered to participate in the study. The couples represented citizenship from Australia, the Philippines, and the United Kingdom.

Each participant voluntarily signed a Research Participant Agreement that provided informed consent, which included the purpose of the study, amount of time necessary and expectations, the right of withdrawal, and protection of anonymity/confidentiality (American Psychological Association, 2017). The participants received no tangible or monetary incentive besides the free access to the online-class materials.

Participants were also asked to provide demographic information (see Table 1 for a summary of demographic data). They identified their marital duration, age, age of children, level of education, language spoken in the home, and life stressors (categories: financial, health, family, legal, expecting a child/child under 1; the additional life-stressor, strict home quarantine, was added later). All considered English as a primary language.

Table 1

Summary of Participant Demographic Variables

\begin{tabular}{lccc}
\hline Demographic variable & $\boldsymbol{M}$ & Range & $\%$ \\
\hline Years married & 7 & $3-16$ & \\
Age & 37 & $32-42$ & $1-2$ \\
Children & 1.7 & $0.5-10$ & 83 \\
Age of children & 4.3 & & 0 \\
Education level & & & 17 \\
Undergraduate or higher & & 0 \\
Some undergraduate/technical & & \\
High school & & \\
Below high school & & \\
\hline Note. $n=6$. Idiosyncratic couple demographics were not provided to protect anonymity. &
\end{tabular}

The couples were living together and were not identifying abuse, addiction, or infidelity in their relationship. However, two couples submitted marital adjustment assessments during Baseline identifying them as distressed.

In addition, the couples indicated a mean of 2.67 major stressors at the time of Baseline but resulted in a mean of four major-stressor categories by the Class phase (FGLO). Table 2 represents the major-stressor categories present for each couple, with related sub-stressors specifically identified by the couples during home lockdown. 
Table 2

Summary of Stressors by Couple at Baseline (BL) and Class (C)

\begin{tabular}{|c|c|c|c|c|c|c|}
\hline \multirow[b]{2}{*}{ Major-stressor category/Sub-stressor } & \multicolumn{2}{|c|}{ Couple 1} & \multicolumn{2}{|c|}{ Couple 2} & \multicolumn{2}{|c|}{ Couple 3} \\
\hline & BL & C & BL & C & BL & C \\
\hline Health & $\mathrm{X}$ & $x$ & $x$ & $x$ & $\mathrm{X}$ & $\mathrm{x}$ \\
\hline Financial & $\mathrm{X}$ & $x$ & $x$ & $x$ & $\mathrm{X}$ & $\mathrm{x}$ \\
\hline Family & $\mathrm{X}$ & $x$ & - & - & - & - \\
\hline Legal & - & - & - & - & - & - \\
\hline Expecting child or child $<1$-year-old & - & - & $x$ & $x$ & - & - \\
\hline Home lockdown (> 2 months) & - & $\mathrm{x}$ & $x$ & $x$ & $\mathrm{X}$ & $x$ \\
\hline Furloughed job without pay & - & $x$ & - & - & - & - \\
\hline Restricted domestic help/childcare & - & $x$ & - & $x$ & - & $x$ \\
\hline Restricted community gatherings & - & $x$ & - & $\mathrm{X}$ & - & $\mathrm{X}$ \\
\hline Work-at-home adjustments & - & $\mathrm{x}$ & - & $\mathrm{X}$ & - & $\mathrm{X}$ \\
\hline School-at-home adjustments & - & - & - & - & - & $x$ \\
\hline
\end{tabular}

At the time of FGLO participation, all three couples experienced a strict home lockdown mandated by the Philippine government for the NCR region. For over 2 months, they were unable to leave their home, except for food and medical needs. School-aged children were immediately home-schooled with online education, if available. Regularly employed domestic staff, such as household or childcare help, were unable to work as all public transportation was halted. Those who were not furloughed needed to make work-at-home adjustments. All community gatherings were not allowed. In summary, the lockdown caused major life disruptions for these couples and necessitated immediate adjustments for work schedule, domestic help, child-care, and home-schooling, besides the underlying fear of contracting COVID-19.

\section{Procedure}

During FGLO, spouses were instructed to complete the self-study assignments for the seven lessons, with some assignments to be completed as a couple. The recommended pace was one lesson per week, requiring about 2-3 hours of homework per lesson. Additionally, there were four, optional booster assignments after FGLO completion.

They also received a weekly, brief telephone check-in (approximately 15 minutes) with this researcher. The check-ins were primarily focused on assignment completion accountability, questions about any problems with technology or assignment links, and topics/assignments for the upcoming lesson. For the lesson and booster on the topic of negotiation, each couple was offered a longer check-in to review the four-step negotiation process learned in the material with a simple, real-life negotiation (two couples accepted the invitations).

Couples also received approximately two, personalized texts and/or brief emails to encourage assignment completion. 


\section{Educational Materials}

FGLO used material from the Four Gifts of Love Participant's Guide (Chalmers \& Harley, 2016) and modified videos from the Four Gifts of Love Class Companion Videos (Chalmers, 2018). The online format was carefully created to provide the same readings, quizzes, and similar activities as the blended-delivery format (FGL; Chalmers, 2019), with the main difference being the weekly, 15-minute, check-ins replacing the weekly, 1.5hour, group sessions.

The main educational purpose of the lessons was to encourage specific behavior change and habit formation to protect the feeling of romantic love with topics based on the marriage concepts of Harley (2010). Participants were taught the concepts and encouraged to apply them through lesson assignments that specifically focused on creating a lifestyle of meeting each other's most important emotional needs in marriage (care), protecting each other from being a source of unhappiness (protection), being transparent with each other (honesty), and scheduling time for undivided attention (time), see Table 3 for a summary of topic details.

Table 3

Marriage Topics by Lesson

\begin{tabular}{|c|c|}
\hline Lesson & Four Gifts of Love class topics \\
\hline 1. Introduction/ FGL review & $\begin{array}{l}\text { Marriage, a life-impacting human relationship; introduction to the Four Gifts of Love; effects of giving } \\
\text { and receiving the gifts of care, protection, honesty and time in marriage; the Love Bank; the Ten Most } \\
\text { Important Emotional Needs in Marriage. }\end{array}$ \\
\hline 2. Care-1 & $\begin{array}{l}\text { Giving and receiving the gift of care; identifying the most important emotional needs; the importance of } \\
\text { listening and thoughtfully responding. }\end{array}$ \\
\hline 3. Care-2 & Sharing emotional needs and starting a plan to meet them for each other. \\
\hline 4. Protection-1 & Giving and receiving the gift of protection; identifying and eliminating Love Busters. \\
\hline 5. Protection-2 & The Policy of Joint Agreement; the Four Guidelines for Successful Negotiation. \\
\hline 6. Honesty & The Five Parts of Honesty; giving and receiving the gift of honesty. \\
\hline 7. Time & The Policy of Undivided Attention; giving and receiving the gift of time. \\
\hline
\end{tabular}

Note. For more details about the skills covered in the Four Gifts of Love Class Online ME intervention, visit

https://www.FourGiftsofLove.org.

There were three to five assignments in each of the seven, online lessons that included articles, quizzes, session videos, and couple-discussion questions. In addition to the marriage-relationship assignments (non-faith based), a faith-based assignment was included in each lesson about using the four main concepts (care, protection, honesty, time) in their relationship with God; if a spouse did not adhere to the beliefs in these lessons, they could choose to only complete the marriage-related assignments. At lessons 4 and 5 , spouses were given specific instructions on giving and receiving information about specific, desired behavior change with an emphasis on listening and thoughtfully responding through individual practice and review of the new behavioral adjustments. In the last lesson, feedback instructions were given to help couples fine-tune their plans of care and protection. 
The website material was mobile-device friendly and included articles, fillable PDF questionnaires, quizzes, and videos (with a closed-caption option) that were static and not necessitating an educator. Vimeo.com was used for viewing the videos that allowed for a lower-quality setting for streaming to accommodate slower internet speed. Google Translate was available on each page if needed. An audio option was added to some of the longer reading assignments for ease of access. The participants were also encouraged to utilize the seven, FGLO mobile apps.

Upon completion of the seven lessons, the couples formally completed FGLO. But they were encouraged to use their online FGLO access to review past assignments and complete four, optional assignments, called "Boosters." Each booster reviewed a major concept from the class (negotiation, care, protection, and impact/legacy). Booster homework included online articles on a previously taught topic (time) and reminders about opportunities to download FGLO mobile apps from the Four Gifts of Love website (www.FourGiftsofLove.org).

\section{Assessment Measures}

All participants individually completed the assessments via a private and secured, online testing link for the duration of the study. Two self-report assessment measures were used to measure marital adjustment and romantic love: the MAT, (see Locke \& Wallace, 1959, to view assessment), and the LBI (see Harley, 2010, to view assessment).

The MAT is a 15-question, self-report survey with variable scoring for each question (potential total score range: 2 to 158) and has been validated as a reliable marital adjustment measure and comparable to more recent, pay-per-use marriage adjustment measures despite its development over 50 years ago (Bagarozzi, 1985; Crane, Allgood, Larson, \& Griffin, 1990; Freeston \& Pléchaty, 1997; Jiang et al., 2013). It is also one of the most cited self-report instruments for marriage research with over 4100 citations across varying cultures and populations (e.g., Ghoroghi, Hassan, \& Baba, 2015; Jiang et al., 2013). The MAT's Spearman-Brown split-half reliability was .90 (Locke \& Wallace, 1959).

The questions focus on general happiness, agreement about marital lifestyle (finances, demonstration of affection, sex relations, philosophy of life), conflict resolution, and leisure time, and specific marital choices (e.g., "If you had your life to live over again, do you think you would: (a) Marry the same person; (b) Marry a different person; or (c) Not marry at all").

Locke and Wallace (1959) defined "adjustment" as "accommodation of a husband and wife to each other at a given time" (p. 251). Crane et al. (1990), and more recently, Whiting and Crane (2003) identified a score of 100 and above as nondistressed or adjusted, 60-99 as moderately distressed, and under 59 as severely distressed.

The LBI is a 21-question, self-report survey with a variable rating (-3: Disagree Completely to +3: Agree Completely) for each question about the spouse. An LBI score is calculated by the sum of ratings for questions 1 through 20 , divided by 20 (potential LBI score range: -3 to +3 ). The Cronbach's alpha was .92 for LBI score and question 21 rating, "I feel romantic love toward (spouse)." This measure has been used for over 35 years across cultures (Harley, 2010). 
Baseline data were collected from three assessment sets for the spouses assigned to the 10-day baseline, and five sets for the spouses assigned to the 2-month baseline with a frequency of approximately every other week submission. Subsequent assessments during FGLO were completed as instructed in the online assignment that began each lesson and at each booster assignment. An email request for follow-up assessments was given at approximately 1 and 2 months after lesson completion.

This study utilized repeated measure submission in an attempted to lessen the potential of a socially desirable response-set bias within the self-report measures. Although research has identified less bias with measures of marital satisfaction constructs (Murstein \& Beck, 1972), the construct of love could have more bias as spouses may believe that one should be in love with a spouse (O'Leary, Acevedo, Aron, Huddy, \& Mashek, 2012). For each online submission, spouses were reminded in writing to respond honestly and individually in an attempt to generate accurate responses.

\section{Data Analysis}

The repeated assessments for each spouse were combined into mean couple scores $(n=3)$. Each mean score was based on submissions of both spouses with no missing data.

The treatment effect was analyzed visually and quantitatively, using both Tau- $U$ (within-case and weighted average; Vannest, Parker, Gonen, \& Adiguzel, 2016) and standardized mean difference (SMD all: within-case; BC-SMD: between-case, Restricted Maximum Likelihood method; Pustejovsky, Chen, \& Hamilton, 2020; Pustejovsky \& Swan, 2018) as recommended for single-case experimental design research (Wolfe, Dickenson, Miller, \& McGrath, 2018; visual analysis: Kazdin, 2011; Tau-U: Parker, Vannest, Davis, \& Sauber, 2011; SMD all: Hedges, Pustejovsky, \& Shadish, 2013; Olive \& Franco, 2008; Olive \& Smith, 2005).

\section{Results}

The mean couple MAT and LBI scores by week are graphically presented in Figure 1 and Figure 2. In addition, the mean scores across Baseline and Class (FGLO) are documented. The gray section identifies the duration of strict home lockdown for the couples. 


\section{Couple 1}

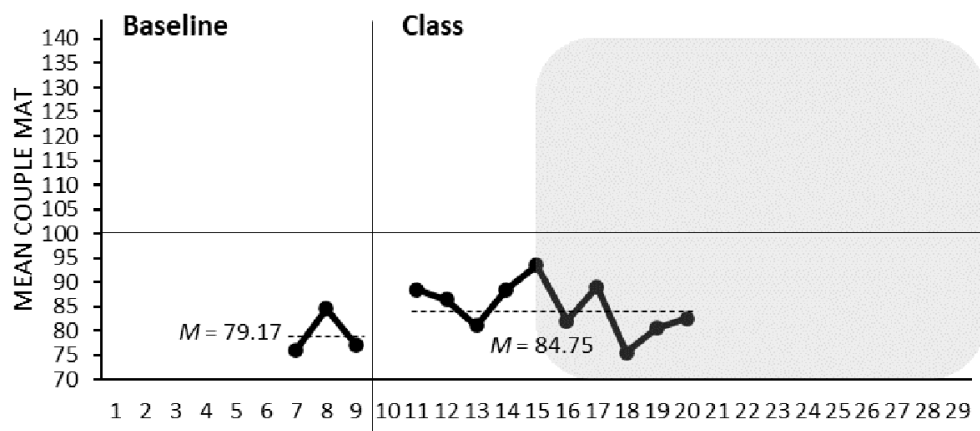

Couple 2

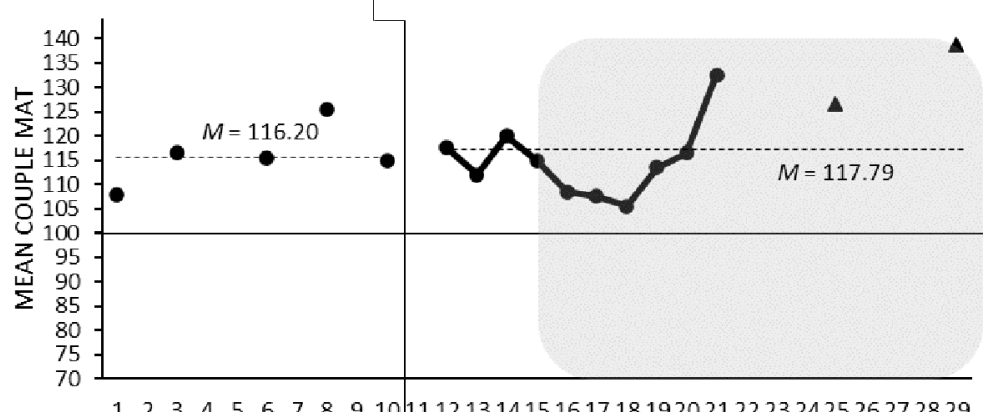

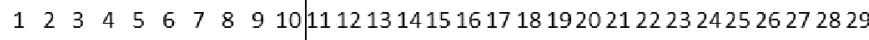

Couple 3

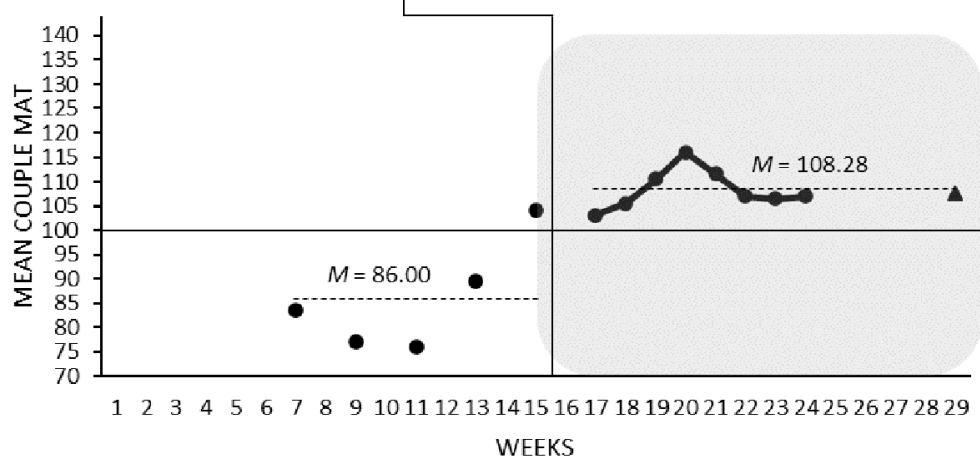

Figure 1. Mean couple MAT by couple for baseline and class phases with mean-trend lines.

Note. Gray portion represents strict home lockdown. The solid line at 100 designates the cut-off score for marital adjustment (>100). $\mathbf{\Lambda}$ : Represents follow-up data, included in analysis. MAT = Marital-Adjustment Test. 


\section{Couple 1}
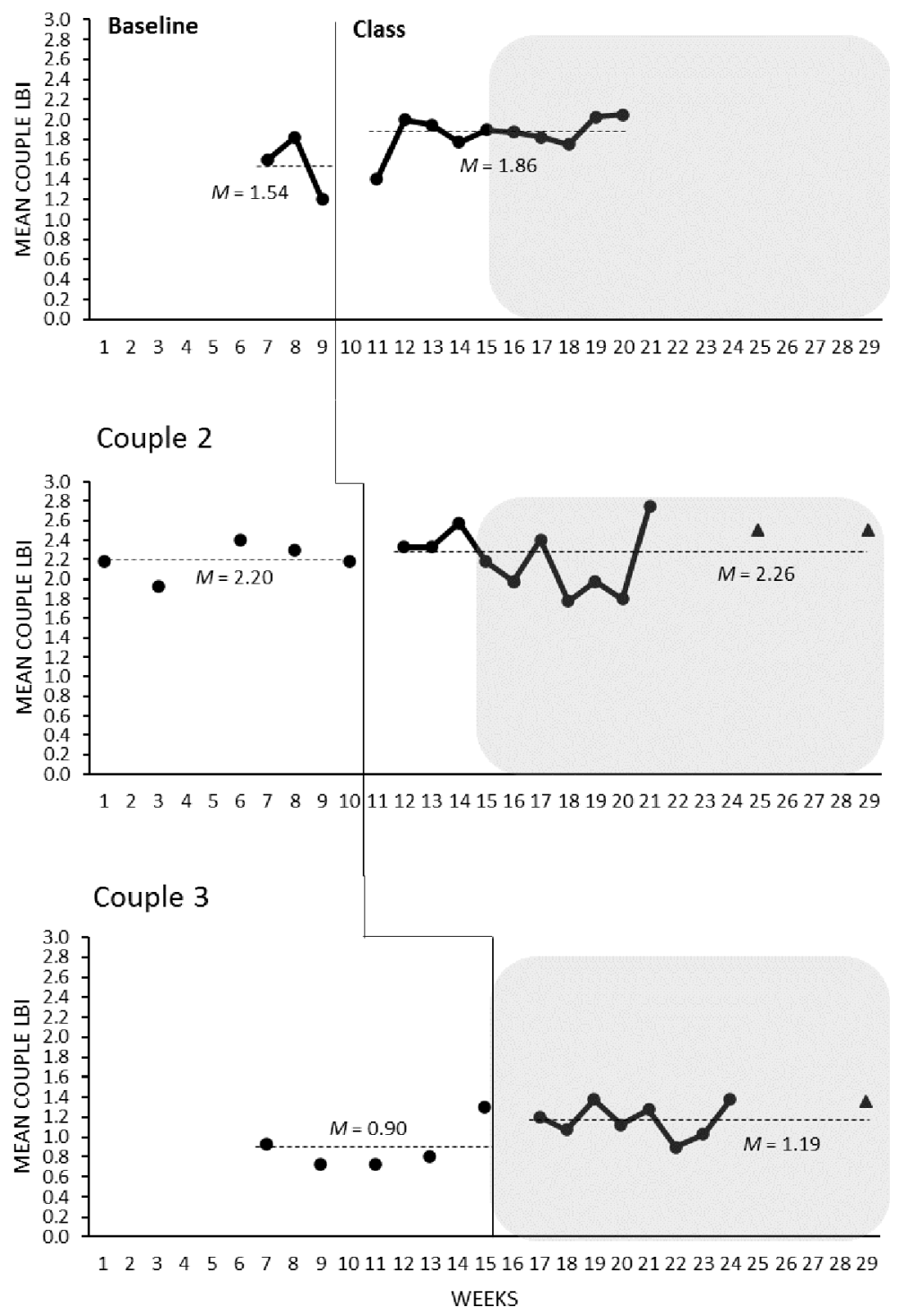

Figure 2. Mean couple LBI by couple for baseline and class phases with mean-trend lines.

Note. Gray portion represents strict home lockdown. $\mathbf{\Lambda}$ : Represents follow-up included in analysis. LBI = Love Bank Inventory.

Regarding the MAT data as seen in Figure 1, varied results were visually demonstrated. Couple 3 showed the greatest gains in marital adjustment scores at the Class phase, while Couple 1 and Couple 2 demonstrated minimal gains. It should be noted that both Couple 1 and Couple 3 had a Baseline MAT mean under the MAT cut off identifying them as moderately distressed (MAT: 60-99; Whiting \& Crane, 2003). Couple 2 was above the cut off for both Baseline and Class, indicating continued stability of marital adjustment during the strict home quarantine.

Table 4 offers the Tau- $U$ and SMD quantitative effect size estimates for MAT with associated analyses. The BC-SMD identified an effect size of 0.34 . The results of the weighted average Tau- $U$ across phases for MAT 
suggested a statistically significant, medium effect size of $.50(p<.05)$. The Tau- $U$ for Couple 3 demonstrated a statistically significant increase in MAT with a large effect size. Although Couple 1 had a Tau- $U$ that would represent a medium effect size, the analysis did not reach statistical significance. Couple 2 demonstrated MAT stability from Baseline to Class phase with an insignificant, but positive effect size.

Table 4

Data Analysis Summary for MAT Across Couples

\begin{tabular}{lccc}
\hline Statistic variable & Couple 1 & Couple 2 & Couple 3 \\
\hline Baseline: $M(S D)$ & $79.17(4.65)$ & $116.10(6.24)$ & $86.00(11.44)$ \\
Class: $M(S D)$ & $84.75(5.33)$ & $117.79(10.14)$ & $108.28(3.83)^{\mathrm{a}}$ \\
Tau- $U\left(S D_{\text {Tau }}\right)$ & $.53(.39)$ & $.03(.32)$ & $.96(.33)$ \\
$\quad p$-value & .176 & .926 & $.004^{* *}$ \\
$90 \% \mathrm{Cl}$ & {$[-.115,1]$} & {$[-.487, .554]$} & {$[.407,1]$} \\
SMD & 0.69 & 0.22 & 1.56 \\
BC-SMD & & 0.34 & .50 \\
Weighted average Tau- $U$ & $.014^{*}$ & \\
$\quad p$-value & & {$[.1651, .8292]$} & \\
$\quad 90 \% \mathrm{Cl}$ & & & \\
\hline
\end{tabular}

Note. $\mathrm{Cl}$ = confidence interval; $\mathrm{SD}$ = standard deviation; SMD = standardized mean difference; MAT = Marital-Adjustment Test; BC = between case. Data for husbands and wives are combined for all measures; each couple is analyzed as a single unit, $n=3$.

aDenotes clinical significance: Class MAT indicating marital adjustment ( $>100)$ from Baseline MAT indicating maladjustment (<100). ${ }^{*} p<.05 .{ }^{* *} p<.01$.

Regarding the effect size for MAT as determined by $S M D_{\text {all }}$, the analysis resulted in a very large effect size estimate for Couple $3(d=1.56)$, a medium effect size for Couple $1(d=0.69)$, and a small effect size for Couple $2(d=0.22)$.

In summary, one couple out of three had clinical and statistical improvements in MAT when phases were compared. Couple 1 showed a medium effect but did not reach clinical significance at the time this study concluded. No follow-up data were received by this couple to identify their trajectory. Couple 2 remained stable for measurements of marital adjustment, during a time of increased distress caused by strict home lockdown for 2 months.

Improvements in LBI data at Class were visibly demonstrated for Couple 3 and Couple 1 as seen in Figure 2. Couple 2 again demonstrated stability as the Class mean remained similar to Baseline. Follow-up data for Couple 2 and Couple 3 suggested stability of measures. No follow-up data for Couple 1 were received.

Table 5 offers the Tau- $U$ and SMD quantitative effect size estimates for LBI with associated analyses. The BC-SMD identified an effect size of 0.31 . The weighted average Tau- $U$ across phases for LBI resulted in a statistically significant, medium effect size of $.52(p<.01)$. The Tau- $U$ for Couple 3 demonstrated a statistically significant increase in LBI with a medium effect size. Although Couple 1 had a Tau- $U$ that would represent a medium effect size, the analysis did not reach statistical significance. Couple 2 demonstrated a slight LBI increase from Baseline to Class phases with a small, nonsignificant effect size. 
Table 5

Data Analysis Summary for LBI Across Couples

\begin{tabular}{|c|c|c|c|}
\hline Statistic variable & Couple 1 & Couple 2 & Couple 3 \\
\hline Baseline: $M(S D)$ & $1.54(0.32)$ & $2.20(0.18)$ & $0.90(0.24)$ \\
\hline Class: $M(S D)$ & $1.86(0.19)$ & $2.26(0.32)$ & $1.19(0.17)$ \\
\hline Tau- $U\left(S D_{\text {Tau }}\right)$ & $.70(.39)$ & $.22(.32)$ & $.69(.33)$ \\
\hline$p$-value & .076 & .493 & $.039^{*}$ \\
\hline $90 \% \mathrm{Cl}$ & {$[.051,1]$} & {$[-.304, .737]$} & {$[.141,1]$} \\
\hline $\mathrm{SMD}_{\text {all }}$ & 0.56 & 0.28 & 0.98 \\
\hline BC-SMD & & 0.31 & \\
\hline Weighted average Tau- $U$ & & .52 & \\
\hline$p$-value & & $.01^{\star *}$ & \\
\hline $90 \% \mathrm{Cl}$ & & {$[.1884, .8525]$} & \\
\hline
\end{tabular}

$\mathrm{SMD}_{\text {all }}$ analyses resulted again in varied effect sizes. Couple 3 had a large effect size estimate, with Couple 1 demonstrating a medium effect size. Couple 2 demonstrated a small effect on LBI measures.

The overall results for LBI would suggest that one couple out of the three (Couple 3) had improvements that reflected a statistically significant, large effect size when comparing Baseline and Class phases. Couple 1 had a medium, nonsignificant effect size. Couple 2 remained stable for measurements of romantic love.

In summary, Couple 3 demonstrated both practical and statistically significant gains in both MAT and LBI when comparing Baseline to FGLO. Couple 1 showed gains that did not reach statistical significance. Couple 2 showed stability for both MAT and LBI during the height of strict home lockdown.

The three couples fully completed the seven lessons and at least one of the four booster assignments after the seven lessons (Boosters completed: Couple 1:3; Couple 2:4; Couple 3:1). There was no attrition.

\section{Discussion}

This study explored the effects of an online ME curriculum on marriage quality with the goal of reducing engagement hurdles. The multiple-baseline design suggested stimulus control over marital adjustment and romantic love measures by FGLO. Visual and quantitative analyses identified improvements in both measures for two couples and stability for one couple. When compared to research evaluating online ME programs conducted during non-calamity circumstances, the effect-size results were similar, but attrition was not.

The limited research of online ME suggested an effect size for various measures of relationship quality that ranged from $d=0.026$ to $d=0.69$, and attrition ranged from 15 to 46\% (Doss et al., 2016; Duncan et al., 2009; Kalinka et al., 2012). Considering that this preliminary study was conducted under intense, world-wide stress as compared to the conditions of these earlier studies, the equivalent effect size results for relationship quality with no attrition are encouraging. The effect size, however, was not as great as the large effect sizes reported by the 
experimental design study for the blended-format FGL, in which FGLO was adapted (Chalmers, 2019; MAT: $d=$ $1.11, p<.01 ; \mathrm{LBI}: d=0.68, p<.05)$.

While the results demonstrated stability and improvements for these couples during a time of calamity, it should also be noted that there were strong gains for two couples who were showing significant distress at baseline (MAT < 100) and could be considered high risk for marital disruption when external stress increases (Bodenmann, 1997). FGLO teaches skills to avoid verbal negativity, express needs with honesty, and dyadically work through problems, which Bodenmann (1997) concluded would help couples who are experiencing stress. After FGLO completion, all four spouses exhibited MAT score increases, with two out of the four reaching scores above 100. This suggests promise for helping distressed couples in times of engagement hurdles and elevated external stress with a flexible-delivery, online ME.

The results were also contrary to the predicted relationship between lower marital satisfaction and elevated COVID-19 related stressors. According to Balzarini et al. (2020), the couples surveyed who experienced social isolation and economic pressure reported significantly lower relationship satisfaction than those not experiencing similar COVID-19 related stressors. They also reported that the prediction of relationship deterioration could be mitigated if partners would thoughtfully respond to each other's needs, which was another focus of FGLO curriculum.

FGLO focuses on helping couples create a lifestyle that meets their spouse's emotional needs, protect from negativity, and come together as a unit to solve problems. The outcome of this study seemed to suggest that this focus may have been successful in mitigating marital deterioration for these three couples who were experiencing social isolation, financial strain, and additional living-related stressors. After completing FGLO, measures of marital satisfaction increased as the couples experienced significant COVID-related stressors.

Although the results are encouraging, there are several limitations and related improvements recommended for future research that should be mentioned. First, the process of being personally invited to join FGLO could have influenced the zero attrition in this study, as compared to the higher attrition of a similar 2-month, online curriculum (46\%: Kalinka et al., 2012) or even a lower-dose, 4-session curriculum (15\%: Doss et al., 2016). The invitation process was used in an attempt to enroll couples who may not typically attend a traditional-delivery marriage class (Morris, McMillan, Duncan, \& Larson, 2011), and it was considered a more organic process for ME class introduction (i.e., via personal referral vs. a non-personal community announcement). The effects of this process on attrition would be an intriguing research topic for future study.

A second limitation involved the communication style used during check-ins and weekly email/texts. This study used a more idiosyncratic- and personal-communication style for the weekly lesson check-ins and encouragement emails/texts from the facilitator, thus lacking standardization. Although this idiosyncratic approach may have been a benefit in lowering attrition, program efficacy could have been altered due to the nonstandardization. An improvement would be to standardize the check-ins and emails/texts to control for any confounding effects of communication style as related to program efficacy. Future research could delve further into program efficacy changes as related to idiocyncratic communication.

Lastly, quantitative stress data would have been valuable in identifying possible considerations for between couple variations. This study only identified major categories of stress, which did not allow for any quantitative analysis of how stress levels may relate to the outcome measures. Bodenmann (1997) would have predicted 
that as stress increases, marital satisfaction would decrease, which is what seemed to happen initially during home lockdown for Couple 1 and Couple 2, with unpredicted increases in marital quality as FGLO was completed. An improvement would be for each spouse to complete a weekly stress assessment in addition to the marital-quality measures.

The goal of this online ME curriculum was to offer an effective, flexible-delivery ME format when a traditional or blended format is unattainable. This exploratory study seemed to suggest that this curriculum was effective in achieving this goal for all three couples as the data represented stability and improvements in marital quality when comparison data from past and present life disruptions would predict a decline (Balzarini et al., 2020; Cohan \& Cole, 2002; Lau et al., 2005; Peacock et al., 1997). In addition, all three couples completed FGLO.

This online-delivery marriage class with a cognitive-behavioral framework that focused on teaching couples how to create and sustain romantic love offers a potential direction for solving engagement hurdles during life disruption. As such, it is recommended that future ME research focus attention on developing online ME as adapted from empirically-validated curriculum to lessen engagement hurdles and reach distressed couples, especially during times of sudden calamity.

\section{Funding}

The author has no funding to report.

\section{Competing Interests}

The author is the co-developer of the Four Gifts of Love Class materials.

\section{Acknowledgments}

The author has no support to report.

\section{Author Note}

This study utilized a similar method and online variation of the marriage education curriculum as in the previously published article, "What's love got to do with it? Improving the effect of marriage education" (Chalmers, 2019).

\section{References}

American Psychological Association. (2017). Ethical principles of psychologists and code of conduct, section 8: Research and publication. Retrieved from https://www.apa.org/ethics/code

Anderson, M. (2019). Mobile technology and home broadband. Pew Research Center. Retrieved from https://www.pewresearch.org/internet/2019/06/13/mobile-technology-and-home-broadband-2019/

Anderson, M., Perrin, A., Jiang, J., \& Kumar, M. (2019). 10\% of Americans don't use the internet. Who are they? Pew Research Center. Retrieved from https://www.pewresearch.org/fact-tank/2019/04/22/some-americans-dont-use-the-internet-who-are-they/

Bagarozzi, D. A. (Ed.). (1985). Family measurement techniques: Locke Marital Adjustment Scale and the Dyadic Adjustment Scale. American Journal of Family Therapy, 13(3), 66-71. https://doi.org/10.1080/01926188508251266 
Balzarini, R. N., Muise, A., Zoppolat, G., Di Bartolomeo, A., Rodrigues, D. L., Alonso-Ferres, M., . . Slatcher, R. B. (2020). Love in the time of Covid: Perceived partner responsiveness buffers people from lower relationship quality associated with Covid-related stressors. PsyArXiv. https://doi.org/10.31234/osf.io/e3fh4

Bodenmann, G. (1997). The influence of stress and coping on close relationships: A two-year longitudinal study. Swiss Journal of Psychology / Schweizerische Zeitschrift für Psychologie / Revue Suisse de Psychologie, 56(3), $156-164$.

Brooks, S. K., Webster, R. K., Smith, L. E., Woodland, L., Wessely, S., Greenberg, N., \& Rubin, G. I. (2020). The psychological impact of quarantine and how to reduce it: Rapid review of the evidence. Lancet, 395, 912-920. https://doi.org/10.1016/S0140-6736(20)30460-8

Census and Statistics Department, Hong Kong. (2018, January). Marriage and divorce trends in Hong Kong, 1991 to 2016. Hong Kong Monthly Digest of Statistics. Retrieved from https://www.censtatd.gov.hk/hkstat/sub/sp160.jsp?productCode=FA100055

Chalmers, J. H. (2018). The Four Gifts of Love class companion video [Prime Video]. Philippines: Magnetic Sphere Production/Stronghold MNL. Retrieved from https://www.amazon.com

Chalmers, J. H. (2019). What's love got to do with it? Improving the effect of marriage education. Interpersona: An International Journal on Personal Relationships, 13(2), 171-186. https://doi.org/10.5964/ijpr.v13i2.361

Chalmers, J. H., \& Harley, W. F., Jr. (2016). The Four Gifts of Love participant's guide: Revised and expanded edition. Marriage Resources International. Retrieved from https://www.amazon.com

Cohan, C. L., \& Cole, S. W. (2002). Life course transitions and natural disaster: Marriage, birth, and divorce following Hurricane Hugo. Journal of Family Psychology, 16(1), 14-25. https://doi.org/10.1037//0893-3200.16.1.14

Cohen, J. (1988). Statistical power analysis for the behavioral sciences (2nd ed.). Hillsdale, NJ, USA: Earlbaum.

Crane, D. R., Allgood, S. M., Larson, J. H., \& Griffin, W. (1990). Assessing marital quality with distressed and nondistressed couples: A comparison and equivalency table of three frequently used measures. Journal of Marriage and Family, 52(1), 87-93. https://doi.org/10.2307/352841

Doss, B. D., Cicila, L. N., Georgia, E. J., Roddy, M. K., Nowlan, K. M., Benson, L. A., \& Christensen, A. (2016). A randomized controlled trial of the web-based OurRelationship Program: Effects on relationship and individual functioning. Journal of Consulting Clinical Psychology, 84(4), 285-296. https://doi.org/10.1037/ccp0000063

Duncan, S. F., Steed, A., \& Needham, C. M. (2009). A comparison evaluation study of web-based and traditional marriage and relationship education. Journal of Couple and Relationship Therapy, 8, 162-180. https://doi.org/10.1080/15332690902813836

Duque, F. T. (2020). Omnibus guidelines on the implementation of community quarantine in the Philippines (Republic of the Philippines Inter-Agency Task Force for the Management of Emerging Infectious Diseases). Official Gazette. Retrieved from https://www.officialgazette.gov.ph/2020/06/25/omnibus-guidelines-on-the-implementation-of-communityquarantine-in-the-philippines-with-amendments-as-of-june-25-2020/

Freeston, M. H., \& Pléchaty, M. (1997). Reconsideration of the Locke-Wallace Marital Adjustment Test: Is it still relevant for the 1990s? Psychological Reports, 81(2), 419-434. https://doi.org/10.2466/pr0.1997.81.2.419 
Georgia, E. J., \& Doss, B. D. (2013). Web-based couple intervention: Do they have a future? Journal of Couple and Relationship Therapy, 12, 168-185. https://doi.org/10.1080/15332691.2013.779101

Ghoroghi, S., Hassan, S. H., \& Baba, M. (2015). Marital adjustment and duration of marriage among postgraduate Iranian students in Malaysia. International Education Studies, 8(2), 50-59. https://doi.org/10.5539/ies.v8n2p50

Harley, W. F., Jr. (2010). Effective marriage counseling. Grand Rapids, MI, USA: Revell.

Hedges, L. V., Pustejovsky, J. E., \& Shadish, W. R. (2013). A standardized mean difference effect size for multiple baseline designs across individuals. Research Synthesis Methods, 4(4), 324-41. https://doi.org/10.1002/jrsm.1086

Jiang, Y., Terhorst, L., Donovan, H. S., Weimer, J. M., Choi, C. W., Schulz, R., . . Sherwood, P. R. (2013). Locke-Wallace Short Marital-Adjustment Test: Psychometric evaluation in caregivers for persons with primary malignant brain tumor. Journal of Nursing Measurement, 21(3), 502-515. https://doi.org/10.1891/1061-3749.21.3.502

Kalinka, C. J., Fincham, F. D., \& Hirsch, A. H. (2012). A randomized clinical trial of online-biblio relationship education for expectant couples. Journal of Family Psychology, 26(1), 159-164. https://doi.org/10.1037/a0026398

Kazdin, A. E. (2011). Single-case research designs: Methods for clinical and applied setting (2nd ed.). New York, NY, USA: Oxford University Press.

Larson, J. H. (2004). Innovations in marriage education: Introduction and challenges. Family Relations, 53(5), $421-424$. https://doi.org/10.1111/j.0197-6664.2004.00049.x

Lau, J. T., Yang, X., Pang, E., Tsui, H. Y., Wong, E., \& Wing, Y. K. (2005). SARS-related perceptions in Hong Kong. Emerging Infectious Diseases, 11(3), 417-424. https://doi.org/10.3201/eid1103.040675

Lenhard, W., \& Lenhard, A. (2016). Calculation of effect sizes. Retrieved from https://www.psychometrica.de/effect_size.html.

Locke, H. J., \& Wallace, K. M. (1959). Short marital adjustment and prediction tests: Their reliability and validity. Marriage and Family Living, 21(3), 251-255. https://doi.org/10.2307/348022

McAllister, S., Duncan, S. F., \& Hawkins, A. J. (2012). Examining the early evidence for self-directed marriage and relationship education: A meta-analytic study. Family Relations: An Interdisciplinary Journal of Applied Family Studies, 61(5), 741-755. https://doi.org/10.1111/j.1741-3729.2012.00736.x

Medialdea, S. C. (2020a). Memorandum from the executive secretary: Community quarantine over the entire Luzon and further guidelines for the management of the coronavirus disease 2019 (COVID-19) situation (Prepared by the Office of the President of the Philippines). Official Gazette.Retrieved from

https://www.officialgazette.gov.ph/2020/03/16/memorandum-from-the-executive-secretary-on-community-quarantineover-the-entire-luzon-and-further-guidelines-for-the-management-of-the-coronavirus-disease-2019-covid-19-situation/

Medialdea, S. C. (2020b). Imposing an enhanced community quarantine in high risk geographic areas of the Philippines and a general community quarantine in the rest of the country from 01 to 05 May 2020, Adopting the omnibus guidelines on the implementation thereof, and for other purposes (Prepared by the Office of the President of the Philippines). Official Gazette. Retrieved from https://www.officialgazette.gov.ph/2020/04/30/executive-order-no-112-s-2020/ 
Morris, M. L., McMillan, H. S., Duncan, S. F., \& Larson, J. H. (2011). Who will attend? Characteristics of couples and individuals in marriage education. Marriage and Family Review, 47(1), 1-22. https://doi.org/10.1080/01494929.2011.558463

Morris, S. B. (2008). Estimating effect size from pretest-posttest-control group designs. Organizational Research Methods, 11(2), 364-386. https://doi.org/10.1177/1094428106291059

Murstein, B. I., \& Beck, G. D. (1972). Person perception, marriage adjustment, and social desirability. Journal of Consulting and Clinical Psychology, 39(3), 396-403. https://doi.org/10.1037/h0033960

National Economic and Development Authority. (2020). Addressing the social and economic impact of the COVID-19 pandemic. GOVPH. Retrieved from http://www.neda.gov.ph/addressing-the-social-economic-of-the-covid-19-pandemic/

O'Leary, K. D., Acevedo, B. P., Aron, A., Huddy, L., \& Mashek, D. (2012). Is long-term love more than a rare phenomenon? If so, what are its correlates? Social Psychological and Personality Science, 3(2), 241-249. https://doi.org/10.1177/1948550611417015

Olive, M. L., \& Franco, J. H. (2008). (Effect) size matters: And so does the calculation. The Behavior Analyst Today, 9(1), 5-10. https://doi.org/10.1037/h0100642

Olive, M. L., \& Smith, B. W. (2005). Effect size calculations and single subject designs. Educational Psychology, 25(2-3), 313-324. https://doi.org/10.1080/0144341042000301238

Parker, R. I., Vannest, K. J., Davis, J. L., \& Sauber, S. B. (2011). Combining non-overlap and trend for single case research: Tau-U. Behavior Therapy, 42(2), 284-299. https://doi.org/10.1016/j.beth.2010.08.006

Peacock, W. G., Morrow, B. H., \& Gladwin, H. (1997). Hurricane Andrew: Ethnicity, gender and the sociology of disasters. New York, NY, USA: Routledge.

Pustejovsky, J. E., Chen, M., \& Hamilton, B. (2020). Scdhlm: A web-based calculator for between-case standardized mean differences (Version 0.5.0) [Web application]. Retrieved from https://jepusto.shinyapps.io/scdhlm

Pustejovsky, J. E., \& Swan, D. M. (2018). Single-case effect size calculator (Version 0.5) [Web application]. Retrieved from https://jepusto.shinyapps.io/SCD-effect-sizes/

Silver, L., \& Huang, C. (2019). In emerging economies, smartphone and social media users have broader social networks. Pew Research Center. Retrieved from https://www.pewresearch.org/internet/2019/08/22/in-emerging-economies-smartphone-and-social-media-users-havebroader-social-networks/

United Nations. (2020). The social impact of COVID-19. Retrieved from https://www.un.org/development/desa/dspd/2020/04/social-impact-of-covid-19/

Vannest, K. J., Parker, R. I., Gonen, O., \& Adiguzel, T. (2016). Single case research: Web based calculators for SCR analysis (Version 2.0) [Web-based application]. College Station, TX, USA: Texas A \& M University. Retrieved from www.singlecaseresearch.org/calculators/tau-u

Whiting, J. B., \& Crane, D. R. (2003). Distress and divorce: Establishing cutoff scores for the Marital Status Inventory. Contemporary Family Therapy, 25(2), 195-205. https://doi.org/10.1023/A:1023672101157 
Wolfe, K., Dickenson, T. S., Miller, B., \& McGrath, K. V. (2018). Comparing visual and statistical analysis of multiple baseline design graphs. Behavior Modification, 43(3), 361-388. https://doi.org/10.1177/0145445518768723 